\title{
2D Reluctance Model of an Eddy Current Brake with a Magneto Isotropic Material Structure
}

\author{
Christoph Holtmann, German Aerospace Centre and Andreas Möckel, TU Ilmenau
}

\begin{abstract}
This work shows the model of an eddy current brake with an magneto isotropic material structure. For the modelling of the magneto isotropic material structure made of steel posts, a reluctance network is recommended, since a full FEM model would require too much computation time for optimization purposes. However, in order to map details like the skin effect in the pins, in the reluctance model some details are modelled with the help of fem and thus the required reluctances are determined. The torque calculations of the model is possible with a deviation of less than $10 \%$ compared to an experiment.
\end{abstract}

Index Terms-brake, eddy current brake, liquid cooled, reluctance model, retarder,

\section{INTRODUCTION}

Conventional eddy current brakes (ECB's) are made of a homogeneous material that moves through an inhomogeneous primary magnetic field at a relative speed $v$ [1] [2]. The resulting eddy currents lead to the displacement of the primary field and thus to the phenomenon of the skin effect [3]. The skin effect is primarily the result of the homogeneous material, in which each infinitely small current is coupled to all others through a magnetic circuit with high permeability. The skin effect can be drastically reduced by interrupting the magnetic circuit in the active material in one direction, so that a conductive material can still be penetrated by the primary magnetic field. This can be realized by using pins that only lead the magnetic field perpendicular to the surface and the pins are surrounded by a conductive material in which the eddy currents occur, as shown in figure I.1 and patent [4]. This method is generally not new, since more or less every electrical machine consists of a magnetically conductive material which is surrounded by an electrically conductive material. However, the pin structure enables a new design for the cooling structure. The basic idea of how to increase the heat-flux in the cooling fluid is to increase the surface of the eddy current material in contact to the fluid. In the ECB with pin structure, the surrounding material can be divided into a number of layers so that the cooling surface is dramatically increased. This method is particularly useful only because the eddy current density in each sheet is nearly the same due to the reduction of the skin effect. The setting of the parameters of the material structure can be compared to a

C. Holtmann is with the Institute of Vehicle Concepts, German Aerospace Centre, Pfaffenwaldring 38-40, 70569 Stuttgart Germany (e-mail: christoph.holtmann@dlr.de).

A. Möckel is with the Department of Electrical Engineering, Technical University Ilmenau, Gustav-Kirchhoff-Str. 1, 98693 Ilmenau Germany (email: andreas.moeckel@tu-ilmenau.de)

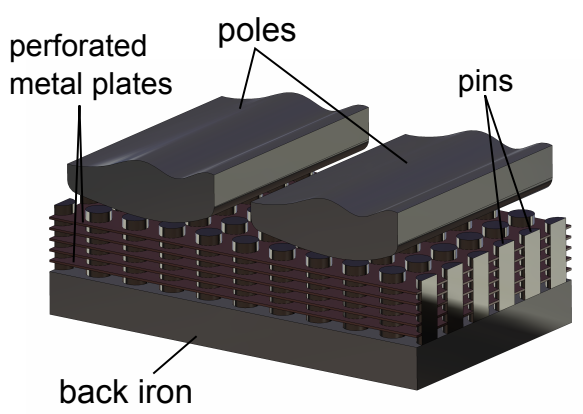

Fig. I.1: basic construction

free parametrization of the conductivity and permeability of a homogeneous material. The average conductivity of the eddy current material for a given total thickness of the structure can be adjusted by the number of sheets and their thickness, while the permeability can be adjusted by the number of pins and their thickness.

In conclusion the advantages of the eddy current brake are

- reducing the skin effect resulting a more homogenous heat generation

- the possibility of increasing the cooling surface

- free material parametrisation for optimising the torque density and torque characteristics

\section{Problem (COMPUtation With FEM)}

Eddy current brakes with homogeneous active material can be calculated using a stationary model, even if they move, by solving Maxwell's equations for moving conductors, as described in [5]. When using the classic FEM method, however, this does not work if the moving conductor has an inhomogeneous structure, so that the Maxwell equations have to be solved for the transient case. If this is done for only one speed, several time steps are required until a quasisteady state is reached. For example, the calculation of the ECB designed in [6] took 2400 time steps until the steady state was reached with a speed of $1000 \mathrm{rpm}$ and an angular step of 0.05 degrees for each time step. This required 19 hours of CPU time with an Intel Core $2 \mathrm{CPU}$ at $2.4 \mathrm{GHz}$ for a 3D-model of one pole with 109906 elements and 34100 nodes to calculate the torque for just one speed. It should be noted that the ECB in [6] could have been calculated using a static model due to the homogeneous active material, but some commercial FEM software is unable to consider the $v \times B$ term in the Maxwell equations for moving conductors $[5]$. 
The active material of the ECB in this work consists of approximately 2000 to 3000 steel pins and the rotor rotates at a maximum speed of approximately $10000 \mathrm{rpm}$ with a number of pole pairs between 5 and 12. During a transient simulation, the rotor should not move over more than a quarter of a pin on the outer circumference per time step. So with a outer diameter of $d_{m}=0.3 \mathrm{~m}$ and a pin diameter of $d_{\text {pin }}=4 \cdot 10^{-3} \mathrm{~m}$ the allowed angle step for one time step should not be greater than 0.7 degrees. Assuming about the same time to reach the steady state condition as in [6] the number of time steps will be about 3000 . Also the mesh at the outer surface region of one pin has to be very fine due to the skin effect. When using a skin mesh, in which the pin is discretized in the radial direction with $N$ shells (see figure III.5(b)), then the inner shell radius of the $n^{\text {th }}$ element is

$$
r_{\text {in }}=\frac{d_{\text {pin }}}{2} \frac{1-e^{-\frac{(n-1) d_{\text {pin }}}{2 N \delta}}}{1-e^{-\frac{d_{\text {pin }}}{2 \delta}}}
$$

and the outer shell radius is the inner radius of the next element $r_{\mathrm{in}+1}$. The number of elements of a pin can be approximated by the sum of the elements over all $N$ shells of the pin. A 3D tetrahedral element of size $r_{\mathrm{in}+1}-r_{\mathrm{in} n}$ has a volume of approximately

$$
V_{\text {tet } n}=\frac{\left(r_{\mathrm{i} n+1}-r_{\mathrm{i} n}\right)^{3}}{12} \sqrt{2} k_{\alpha}
$$

where $k_{\alpha}$ is a factor that takes into account that some angles between element edges are smaller than $60^{\circ}$. For this reason, an element is up to four times larger, and a factor of $k_{\alpha}=4$ is used. Every shell $n$ with the volume $V_{\mathrm{sh} n}$ of a half pin includes

$$
N_{3 \mathrm{D} n}=\frac{V_{\mathrm{sh} n}}{V_{\mathrm{tet} n}}=\frac{\pi\left(r_{\mathrm{i} n+1}^{2}-r_{\mathrm{i} n}^{2}\right) L_{\mathrm{pin}} / 2}{V_{\mathrm{tet} n}}
$$

3D elements. Where $L_{\text {pin }} / 2$ is the length of one half pin due to model symmetry. Finally the total Number of 3D tetrahedral elements of a pin is

$$
N_{3 \mathrm{D}}=\sum_{n=1}^{n=N} \frac{\pi\left(r_{\mathrm{i} n+1}^{2}-r_{\mathrm{in}}^{2}\right) L_{\mathrm{pin}} / 2}{V_{\mathrm{tet} n}}
$$

In a mesh study of the pin sub-model in section III the $L_{2}$ error for different numbers of mesh layers in radial direction is approximated for different frequencies and thus for different skin depths. The following figure II.1 shows the resulting relative $L_{2}$ error of flux density for different numbers of radial layers compared to 25 layers. With a frequency of $f=116.6 \mathrm{~Hz}$ and a skin depth of $\delta=2.3 \cdot 10^{-3} \mathrm{~m}$, there is a relative error of less than $10 \%$ after 7 radial layers. This number of layers together with the skin depth of $\delta=2.3 \cdot 10^{-3}$ meter results in approximately $N_{3 D}=7000$ $3 \mathrm{D}$ tetrahedral elements for a pin and in a minimal number 5 million elements for a $60{ }^{\circ}$ segment model of the ECB. At a frequency of $f=1166.6 \mathrm{~Hz}\left(n=10000 \mathrm{~min}^{-1}, p=7\right)$ the error is less than $10 \%$ after 11 radial layers. With a skin depth of $\delta=0.7 \cdot 10^{-3} \mathrm{~m}$ at this frequency, this results in a number of $N_{3 D}=156 \cdot 10^{3} 3 \mathrm{D}$ tetrahedral elements for a pin. So the number of elements in a $60^{\circ} 3 \mathrm{D}$ model is about $50 \cdot 10^{6}$. With this number of elements and 3000 time steps, the calculation for just one speed on a conventional PC, as used in [6], takes several 1000 hours. With adequate resources, this time can potentially be reduced by a factor of 100 , which results in several 10 to 100 hours for a speed, and it takes weeks to calculate the torque speed curve for a design. It must be pointed out that the network analyses are carried out with first order elements. A model with second-order elements requires a smaller number of elements with the same accuracy, but more computing time relative to the number of elements compared to first-order elements. The number of elements is also an optimistic approximation, which only contains the elements of the pins and only corresponds to a segment model of $60^{\circ}$. Most pole pairs require a full model or a half segment model with 3 or 6 times more elements. 3D FEM is therefore not helpful for optimizing this ECB.

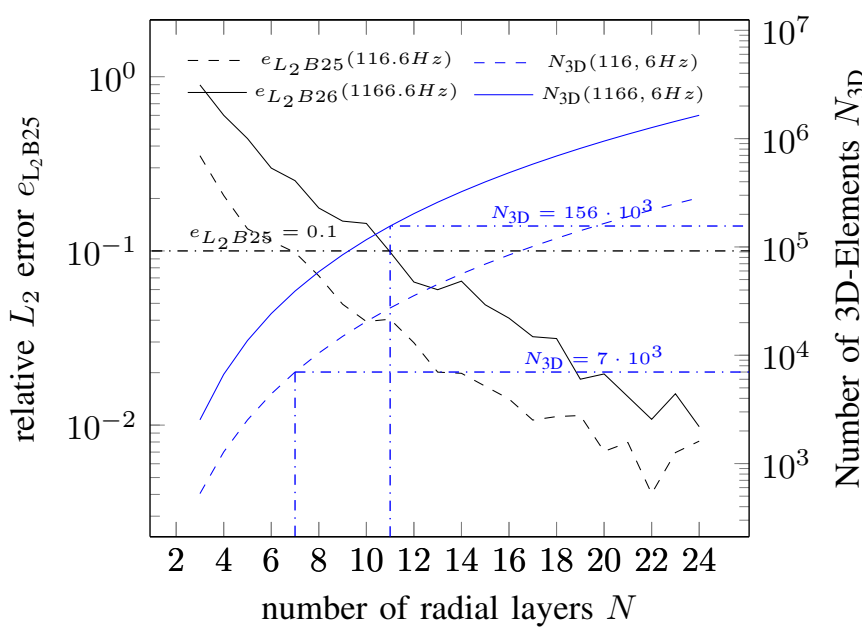

Fig. II.1: Relative $L_{2}$ error of the flux density and number of tetrahedral 3D elements of one pin for different numbers of radial mesh layers at a frequency of $f=116.6 \mathrm{~Hz}$ and $1166.6 \mathrm{~Hz}$.

\section{MODEL}

\section{A. Modelling Method}

The main goal of the model is to compute the ECB's torque speed curve for a given geometry and excitation current. The average torque $\bar{M}$ over one revolution at constant angular speed $\omega$, constant excitation current $I_{\mathrm{ex}}$ and constant temperature of an ECB can be determined from the energy balance of the ECB, including the mechanical power $\bar{M} \omega$, the average ohmic losses in the active material as a result of the eddy currents $\bar{P}_{\text {eddy }}$, the average hysteresis losses $\bar{P}_{\text {hyst }}$ and the mechanical friction losses $\bar{P}_{\text {fric }}$. In the ECB of this work, the hysteresis losses are only taken into account in the pins and mixed with eddy current losses in the pins to the pin iron losses $\bar{P}_{\text {piron }}$.

The model is based on the following assumptions and simplifications 
- the model is a reluctance-network

- the magnetic circuit is modelled two-dimensionally in a pole cross section at the mean effective radius in order to calculate the mean eddy current across all pin columns in the radial direction.

In order to model the details such as the field repression in the steel pins, the reluctance model contains the results of three sub-models. In the first, the pin model, the complex average relative permeability of a pin is calculated depending on the field strength and the frequency. The second model is used to calculate air reluctances and the third to calculate the electrical resistance of the eddy current path between two pins.

\section{B. Geometry}

The ECB is a rotating double-sided axial flux ECB with an outer diameter of $d_{o}$ and the length $l_{\mathrm{ecb}}$, as shown in III.1. The two rotors consist of $2 p$ poles with excitation windings and the back iron. The stator with an isotropic material structure, as shown in Figure I.1, is located between the two rotors. Figure III.2 shows the geometric parameters of the poles and the isotropic material structure according to detail (a) in Figure III.1.

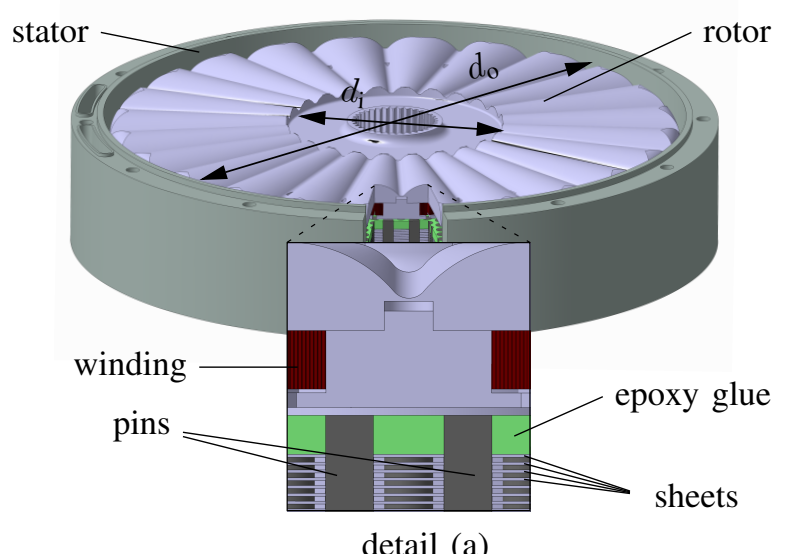

Fig. III.1: CAD model of the rotational axial flux ECB: Detail (a) shows one half pole cross section in the tangential-axial plane at the middle effective radius corresponding to III.2

The isotropic material structure consists of $N_{s}$ perforated sheets with a thickness of $s$, which are penetrated by a large number of steel pins with a diameter of $d_{\mathrm{p}}$. Coolant flows between the sheets in channels of thickness $s_{\mathrm{c}}=\Delta s$ and the structure is fixed and sealed with epoxy adhesive of thickness $h_{\text {ep. }}$ In practice, the pins are arranged in the radial plane, as shown in Figure III.3(a), which corresponds to figure IV.1.

In the model, the real pin pattern is converted into a rectangular model pin pattern, which is shown in III.3(b). Assuming a homogeneous magnetic tension, the 2D reluctance model refers to the mean diameter $d_{\mathrm{m}}$, whereby the inner and outer planar planes are the same, so that the force of the inner and outer planes are also the same.

$$
d_{\mathrm{m}}=\sqrt{\frac{d_{\mathrm{o}}^{2}+d_{\mathrm{i}}^{2}}{2}}
$$

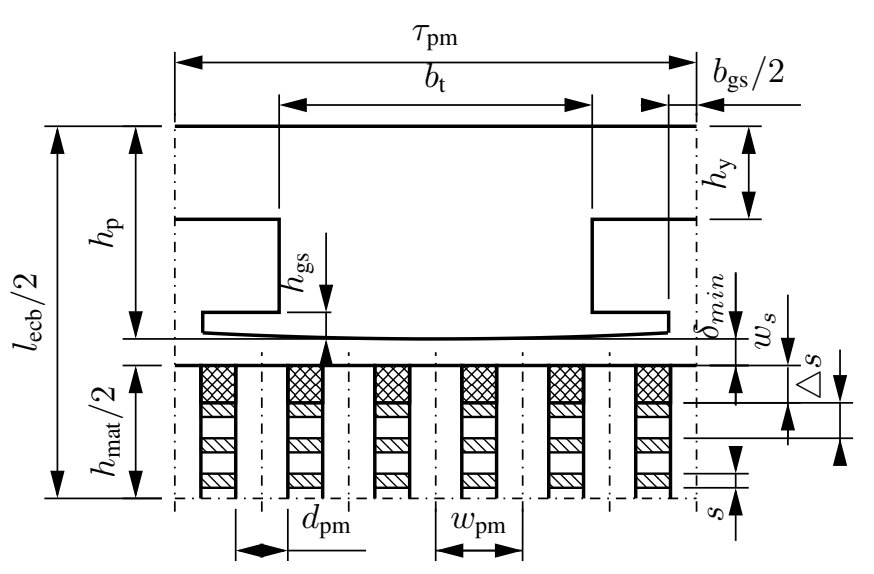

Fig. III.2: Geometrical parameters of one pole cross section

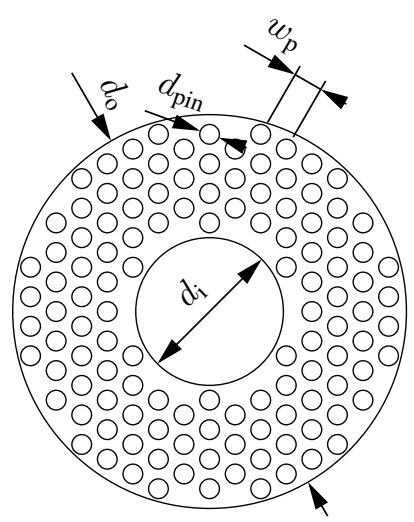

(a) real pin pattern

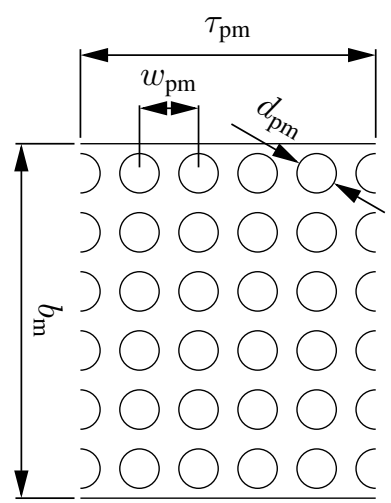

(b) model pin pattern
Fig. III.3: Transformation from the real pin pattern parameters, corresponding to figure IV.1 to the model pin pattern parameters.

The resulting model pole pitch $\tau_{\mathrm{pm}}$ in figure III.3(b) refers to the average diameter $d_{\mathrm{m}}$ and the total area of the structure in the model must match the real surface. Hence the width of the model structure is

$$
b_{\mathrm{m}}=\frac{\pi\left(d_{\mathrm{o}}^{2}-d_{\mathrm{i}}^{2}\right)}{8 p \tau_{\mathrm{pm}}}
$$

The model is calculated with an integer number of pins under one pole $N_{\tau m}$ with a resulting model pin distance $w_{p m}$.

$$
N_{\tau m}=\left\lfloor\sqrt{\frac{k_{p} 4 \tau_{\mathrm{pm}}^{2}}{\pi d_{p}^{2}}}\right\rfloor \quad ; \quad w_{p m}=\frac{\tau_{\mathrm{pm}}}{N_{\tau m}}
$$

\section{Reluctance Network an Equation System}

Figure III.4 shows the reluctance network with $N_{\tau \mathrm{m}}$ pins in one pole pitch $\tau_{\mathrm{pm}}$. The excitation current $I_{e x}$ and the eddy 
currents $i_{i, j}$ in the material structure causing a magnetic flux $\phi_{i, j}$ in each pin segment, where $i$ is the pin index in the tangential (movement) direction and $j$ is the index of the pin segment with the length $\triangle s$ (see right side in figure III.2) in axial direction.

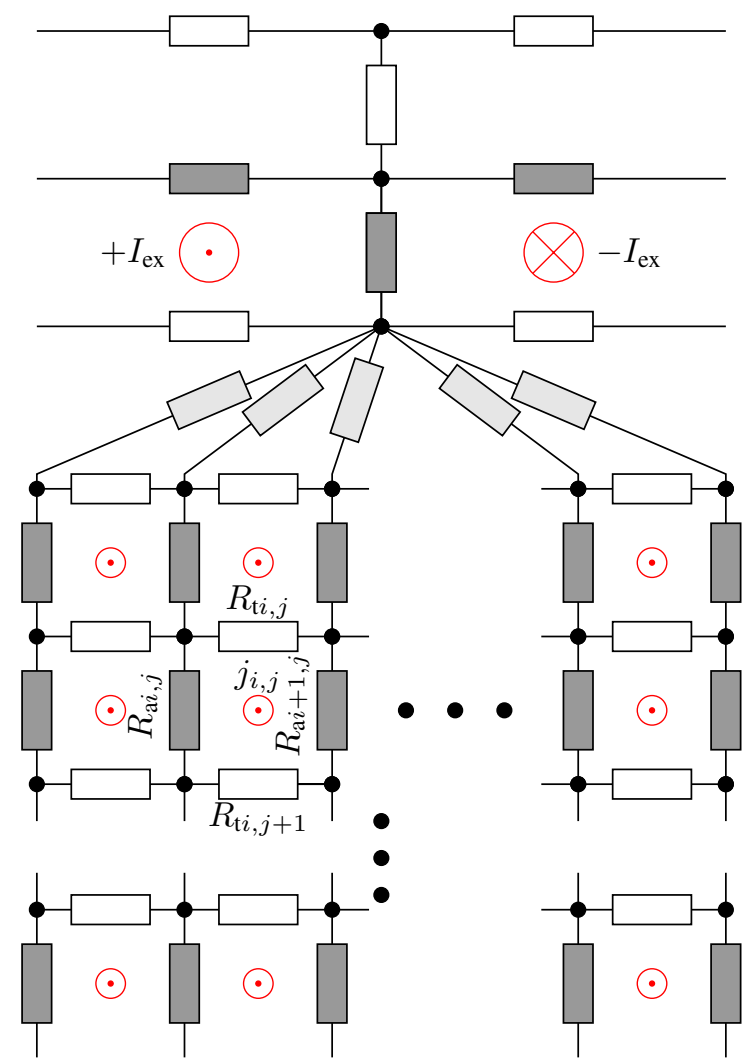

Fig. III.4: 2-D reluctance network. Air reluctances white, steel reluctances grey, and airgap reluctances light grey cause they include the pin reluctances next to the airgap.

The reluctance network results in the mesh equation

$$
\begin{aligned}
& \left(\sum R_{\mathrm{ijn}}\right) \phi_{\mathrm{m} i, j}-\sum\left(\phi_{\mathrm{m} i j n} R_{\mathrm{i}, \mathrm{j}, \mathrm{n}}\right)= \\
& =-\frac{\phi_{\mathrm{m}(i+1, j)}-\phi_{\mathrm{m}(i-1, j)}}{R_{\mathrm{e}} 2 w_{\mathrm{pm}}} v+I_{\mathrm{ex}}
\end{aligned}
$$

where $\phi_{\mathrm{m} i j}$ is mesh flux of the mesh $i j$ which is the analogy to the vector potential $A$ in the transient magnetic field diffusion equation [5]. Further $R_{i j n}$ are the path reluctances of one mesh $i j$ to neighboured meshes with the flux $\phi_{\text {mijn }}$. Corresponding to figure III. 4 the sum of the Reluctances in the first therm is

$$
\sum R_{\mathrm{ijn}}=R_{\mathrm{t} i, j}+R_{\mathrm{t} i, j+1}+R_{\mathrm{a} i, j}+R_{\mathrm{a} i+1, j}
$$

$R_{\mathrm{e}}$ is the electric resistance of one eddy current path and $I_{\mathrm{ex}}$ is the external applied excitation current which only exists in the meshes with excitation windings. The first term on the right represents the eddy currents $j_{i j}$ as a result of the mesh flux change and is the outcome of applying the chain rule to the flux change over time due to the speed of the rotor $v$ at the mean effective radius and the discretization by central difference, where $w_{\mathrm{pm}}$ is the distance between two adjacent pins in the direction of movement.

$$
\frac{\partial \phi_{\mathrm{m}}}{\partial t}=\frac{\partial \phi_{\mathrm{m}}}{\partial x} \frac{\partial x}{\partial t}=\frac{\partial \phi_{\mathrm{m}}}{\partial x} v \approx \frac{\phi_{\mathrm{m} i+1, j}-\phi_{\mathrm{m} i-1, j}}{2 w_{\mathrm{pm}}} v
$$

To transform the equation (8) into a system of equations that can be solved numerically, the first therm of the right side is turned to the left, and some entries of the resulting system matrix contain $v /\left(R_{\mathrm{e}} 2 w_{\mathrm{pm}}\right)$.

\section{Pin Reluctances}

Since the steel pins in the reluctance network are modelled as flux tubes with a homogeneous magnetic flux density, the field repression in the pins is considered by an average relative permeability as a function of the amplitude of the field strength $\hat{H}$ and the frequency as a result of a harmonic analysis. Due to the harmonic analyses, the average relative permeability is complex and is defined with

$$
\underline{\mu}_{\mathrm{r}}=\frac{4 \underline{\phi}_{\mathrm{pm}}}{\hat{H} \mu_{0} \pi d_{\mathrm{p}}^{2}}
$$

where $\phi_{\mathrm{pm}}$ is resulting total complex magnetic flux in the pin. An analytic solution of the field repression in a solid cylinder of constant permeability in a axial time harmonic field is given in [7]. However because of the non linear material behaviour of the pins, the total magnetic flux as a function of the frequency and the magnitude of the field strength $\hat{H}$ is evaluated with the following FEM model. The FEM model is a quasi-static harmonic model that uses the method in [8] to account for non-linearities in the harmonic analysis. Due to the rotational symmetry, a pin is modelled in 2D in the radial axial plane $(r, z)$, as shown in Figure III.5(a). Without showing it here, the result is a classic characteristic of the complex middle relative permeability as a function of the frequency for different field strength amplitudes as shown in [9]. In order to estimate the necessary number of elements for a 3D-model, the 2D model is also used for a mesh study in which the $L_{2}$ error [10] is determined for a different number of radial layers $N$. Figure III.6 shows the real part of the flux density over the radius $r$ of the pin at a frequency of $f=1166 \mathrm{~Hz}$ for different numbers of radial network layers. It can be seen that the result, with increasing number of radial layers, approaches that with $N=25$ radial layers, which comes very close to the exact solution. The $L_{2}$ error norm for $N=1 . .24$ is approximated with the result of the flux density $B$ with $N=25$ layers with

$$
e_{L_{2} B 25}=\frac{\int_{\Omega}\left(B_{25}-B_{\mathrm{N}}\right)^{2} \mathrm{~d} \Omega}{\int_{\Omega} B_{25}^{2} \mathrm{~d} \Omega}
$$

where $\Omega$ is the calculation domain. The result of the error mesh study was already shown in figure II.1 in section II. 


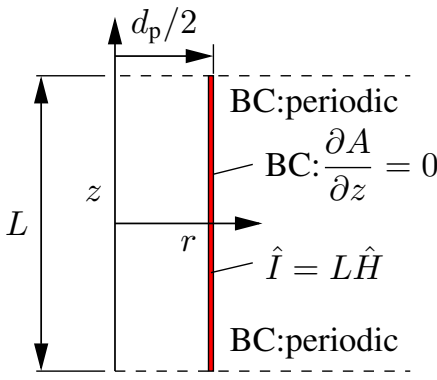

(a) FEM-Model of the pin

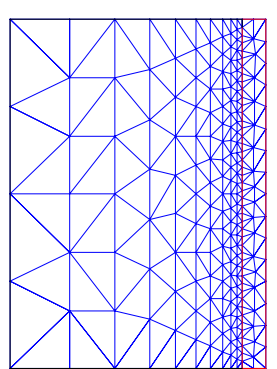

(b) mesh with $N=10$
Fig. III.5: 2D FEM model of one pin. Model with boundary conditions (BC) left and right mesh with $N=10$ radial layers corresponding to equation (1).

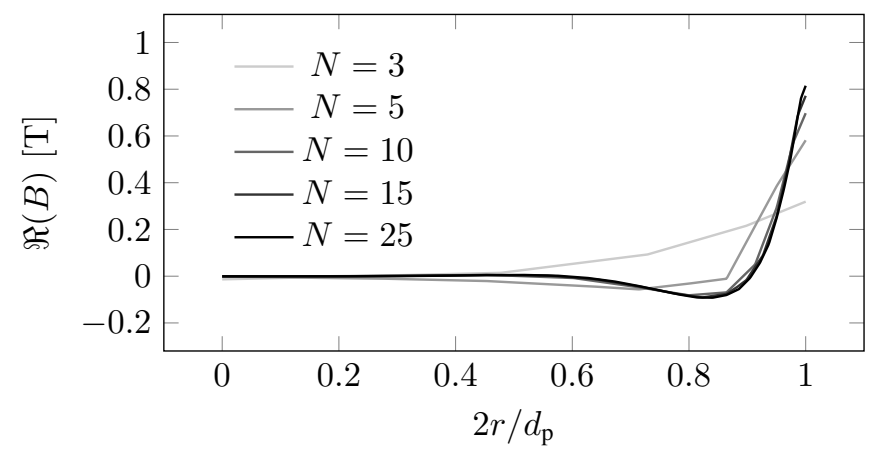

Fig. III.6: Real part of the flux density in one pin at $f=1166 \mathrm{~Hz}$ for different numbers of radial mesh layers $N$.

\section{E. Air Reluctances and Path Resistance}

1) Resistance of the Eddy Current Path: The resistance of the eddy current path between two pins depends on the sheet thickness $s$, the conductivity $\sigma$ and the pin fill factor $k_{p}$. Since the model refers to the middle row of pins, the resistance of the eddy current paths $R_{e}$ in equation (8) is

$$
R_{e}=\frac{1}{\sigma_{\mathrm{s}} k_{\mathrm{n}} s} R^{*}\left(k_{\mathrm{p}}\right)
$$

where $R^{*}$ is a dimensionless resistance factor due to the barrier of the pins and $k_{\mathrm{n}}$ is the Russell and Norsworthy factor [11]. The resistance factor is calculated with the 2DFEM model shown in III.7(b). It is the smallest model to compare the resistance of a perforated sheet with the pin filling factor $k_{\mathrm{p}}$ to a non perforated sheet. A non perforated sheet with $k_{p}=0$ in this model has a resistance of

$$
R_{\mathrm{ekp} 0}=\frac{\sqrt{\frac{3}{4}}}{0.5 s \sigma_{\mathrm{s}}}
$$

With the potential difference of $\triangle \phi=1 \mathrm{~V}$ (see the potential boundary's $\phi=1 \mathrm{~V}$ and $\phi=0 \mathrm{~V}$ in figure III.7(b) ) the value of the resulting total current at the boundary with $\phi=$ $0 \mathrm{~V}$ or $\phi=1 \mathrm{~V}$ automatically will be the inverse value of the resistance $R_{\mathrm{e}}$. The division of the so evaluated resistance with the resistance of the non perforated sheet result in the

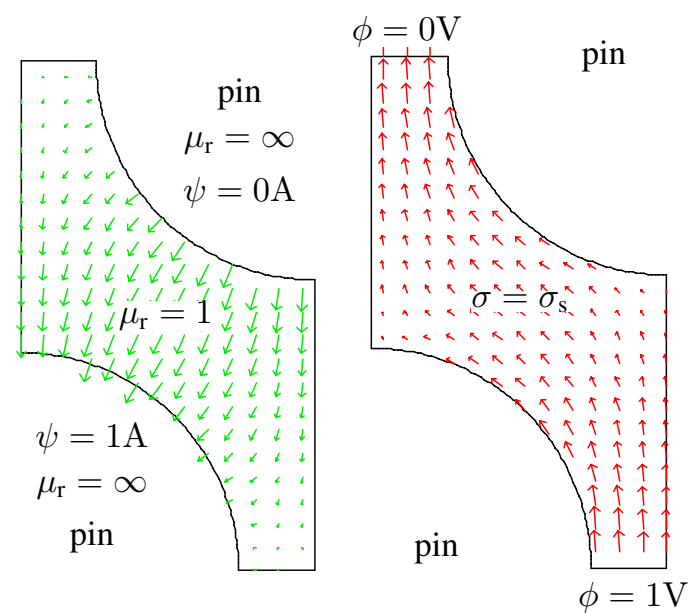

(a) FEM model neighboured (b) FEM modell path resistance reluctance

Fig. III.7: FEM models to calculate the path resistance of the eddy currents (a) and the reluctance between neighboured pins (b). All walls with zero normal flux or current $\mathrm{BC}$ except of those with the boundary conditions shown in the picture.

dimensionless resistance factor

$$
R_{\mathrm{e}}^{*}=\frac{R_{\mathrm{e}}}{R_{\mathrm{ekp} 0}}
$$

which is shown in figure III.8 as a function of the pin filling factor with the solid red line. For a pin filling factor of $k_{p}=0$ the resistance factor is $R^{*}=1$. The Russell and Norsworthy factor $k_{\mathrm{n}}$ [11] is a correction factor that takes into account the finite width $b_{m}$, which would have to be infinite for an exact solution of the $2 \mathrm{D}$ model. With the parameters in this work $k_{\mathrm{n}}$ is

$$
k_{\mathrm{n}}=1-\frac{2 \tau_{\mathrm{pm}}}{\pi b_{\mathrm{m}}} \tanh \left(\frac{\pi b_{\mathrm{m}}}{2 \tau_{\mathrm{pm}}}\right) .
$$

2) Neighboured Pin Air Reluctance: Since the reluctance between the pins is the same with or without eddy currents in the sheets, it can be calculated by solving the Laplace equation for the scalar magnetic potential $\psi$ that can be applied in current free areas [12]. Therefore, the reluctance between pins in the tangential direction is calculated using the model shown in Figure III.7(a). As a boundary condition, a scalar magnetic potential $\psi$ is applied to each boundary of the pins. As with the path resistance calculation, the reluctance depends only on the length of a pin element $\Delta s$ and the pin fill factor, and the potential difference is set to one. Therefore, the value of the reluctance between adjacent pins is the reverse value of the magnetic flux between the pins. Hence the reluctance factor $R_{\mathrm{mnp}}^{*}$ is

$$
R_{\mathrm{mnp}}^{*}=\frac{R_{\mathrm{mnp}}}{R_{\mathrm{mnp} 0}}=R_{\mathrm{mnp}} \mu_{0} \Delta s
$$

3) Air Gap Reluctance: The reluctance of the air gap depends primarily on the air gap $\delta$ and the cross section of a pin. Due to flux fringing at the edges of the pin, the resulting 


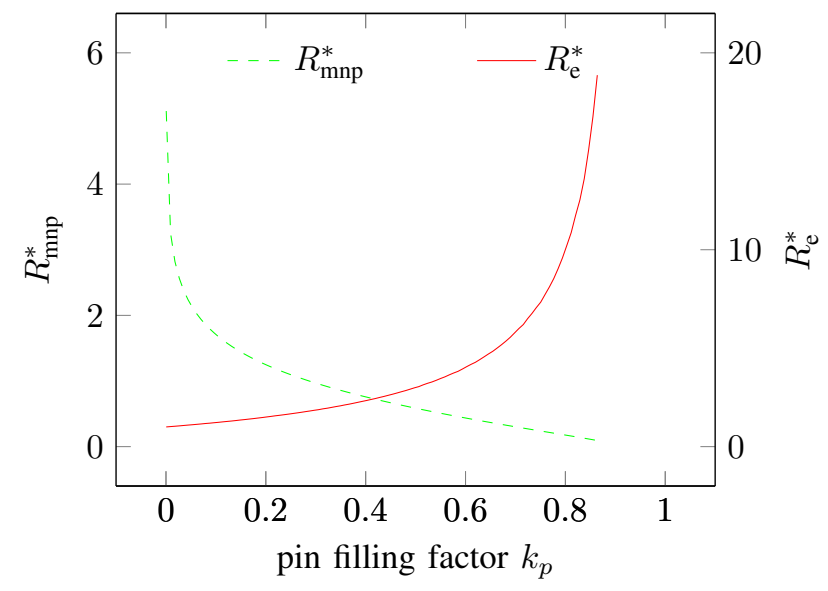

Fig. III.8: Dimensionless resistance of the eddy current path $R_{\mathrm{e}}^{*}$ and dimensionless reluctance between adjacent pins as a function of the pin filling factor $k_{p}$.

reluctance is multiplied by an air gap reluctance factor $k_{\mathrm{m} \delta}$.

$$
R_{\mathrm{m} \delta}=R_{\mathrm{m} \delta 0} k_{\mathrm{m} \delta}=\frac{4 \delta}{\mu_{0} \pi d_{\mathrm{p}}^{2}} k_{\mathrm{m} \delta}
$$

The air gap reluctance factor is calculated using the FEM model in figure III.9, with the air gap reluctance being determined in the same way as the adjacent pin reluctance or path resistance.

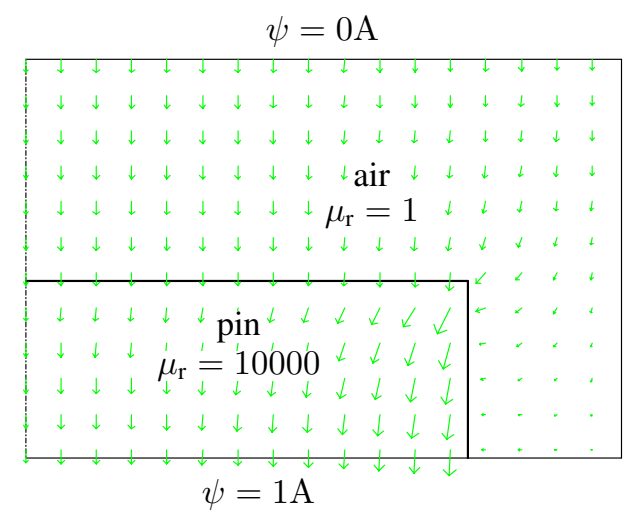

Fig. III.9: FEM-Models to calculate the air gap reluctance. All walls with zero normal flux boundary conditions, except of those with the boundary conditions shown in the picture.

Without eddy currents, there is no flow from one pin to an adjacent pin directly under a pole. So there is a normal boundary condition around a pin with zero flux density to the neighbouring pins in a $60^{\circ}$ pattern. This pattern corresponds to a circle to a good approximation and the reluctance of the air gap is calculated in the cylindrical model in 2D with the boundary conditions shown in figure III.9. The result is displayed in III.10. With high pin fill factors $k_{\mathrm{p}}$, the flux fringing becomes smaller and the air gap reluctance factor for all air gaps is close to one. With small pin fill factors, the flux fringing increases with increasing air gap and therefore the air gap reluctance factor decreases.

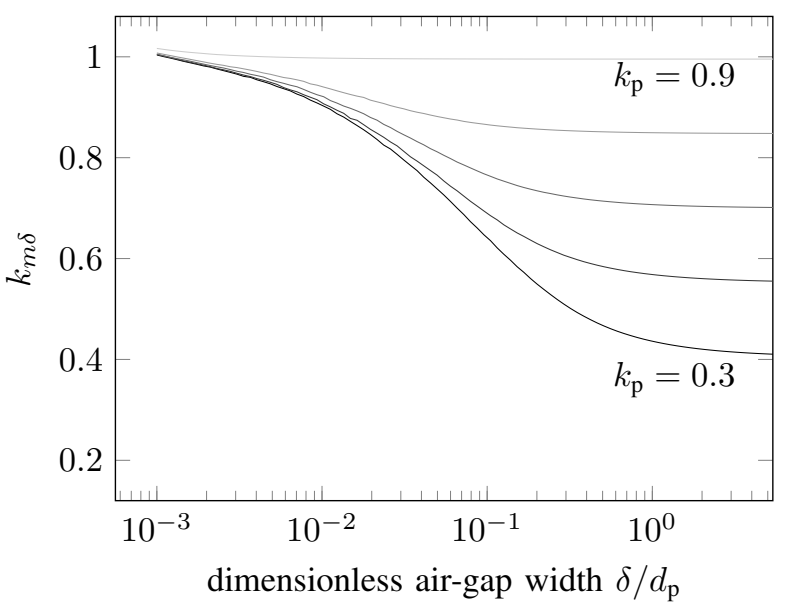

Fig. III.10: Air gap reluctance factor $k_{m \delta}$ as a function of the dimensionless air gap $\delta^{*}=\delta / d_{\mathrm{p}}$ for different pin filling factors $k_{\mathrm{p}}$, calculated with the FEM model in shown in figure III.9.

\section{EXPERIMENTAL VALIDATION}

To validate the $2 \mathrm{D}$ reluctance model, a magneto isotropic material structure, as described in this work, is built into a test $\mathrm{ECB}$, as shown in IV.1. The torque-speed curve is measured on a test bench at constant speeds up to $n=2500 \mathrm{~min}^{-1}$ for various excitation currents, as shown in figure IV.2 with the marked dash-dotted lines. In addition, the mean magnetic flux density is measured in a pin at the air gap next to the mean effective diameter using a flux probe. During the measurement the material structure is cooled with water and the temperature at about $50^{\circ}$ to $80^{\circ}$.

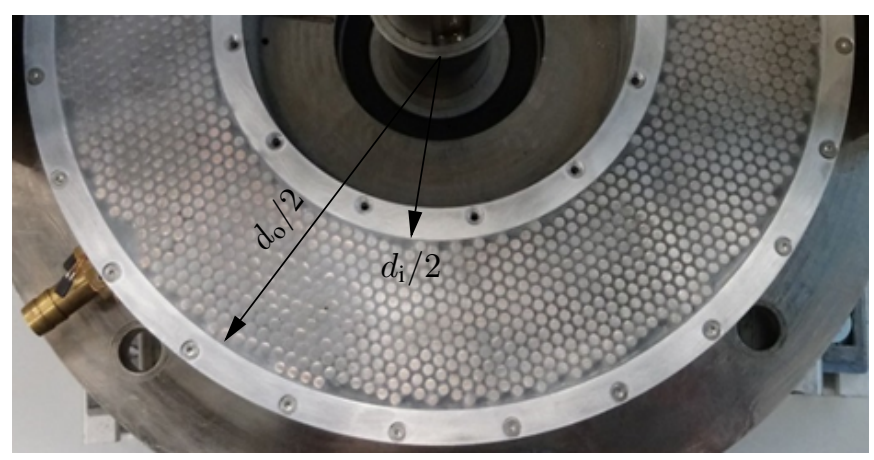

Fig. IV.1: Pin structure in the test ECB corresponding to figure III.3(a)

In figure IV.2 it can be seen that the predicted torque curves (solid lines) are very close to the measured torque curves. The maximum deviation is less than $10 \%$. Figure IV. 3 shows the measured and predicted flux density over a pole distance $\tau_{\mathrm{p}}$. For low frequencies, the flux density in the top of a pin is also predictable with a small error. With increasing speed, the average error over a pole pitch increases to $20 \%$ at $n=2500 \mathrm{~min}^{-1}$. The error is likely to result from the fact that the field repression in the pins also effects the air gap reluctance, hence the error depends on the frequency. 


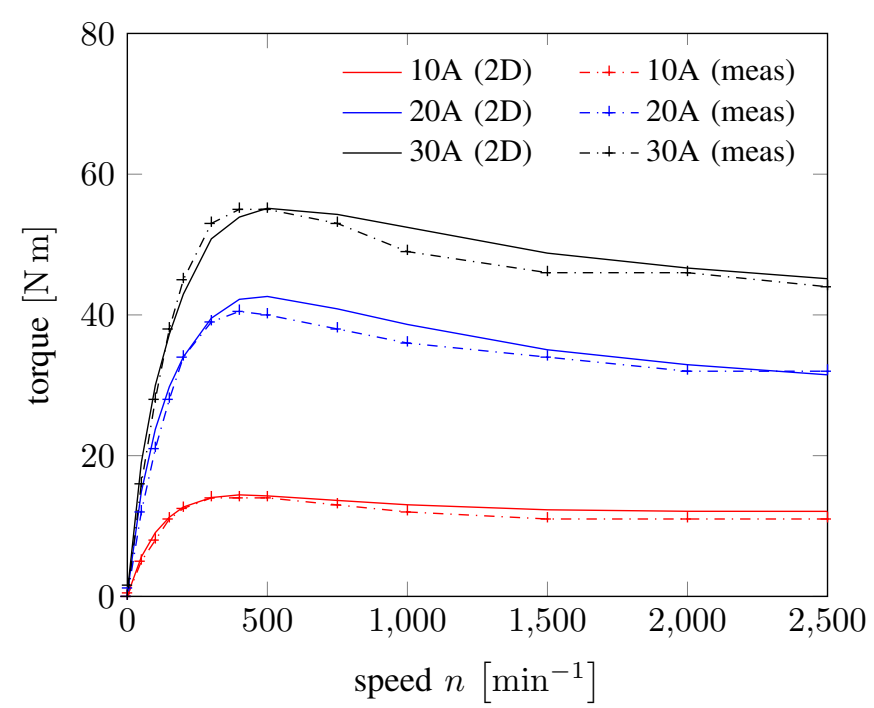

Fig. IV.2: Predicted and measured torque speed curves for different excitation currents

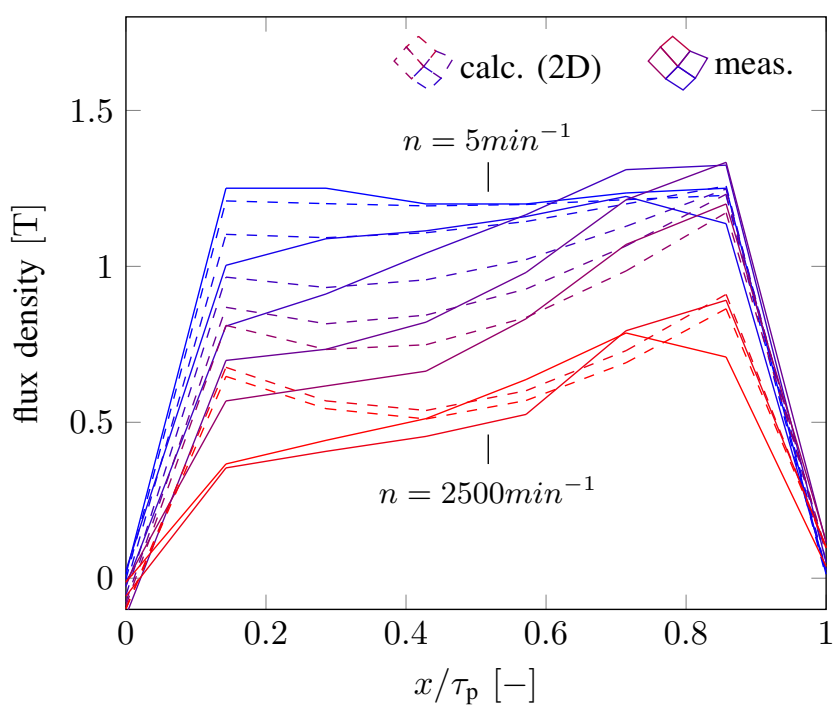

Fig. IV.3: Predicted and measured flux density in the top of one pin next to the air gap for different speeds $n$

\section{CONCLUSION}

In this work it was shown why a reluctance network is required to calculate this type of ECB. Using a 3D-FEM model it would take weeks to months to calculate the torquespeed curve for just one design. The $2 \mathrm{D}$ reluctance network enables the calculation of the torque-speed curve with 12 data points with an error of less than $10 \%$ in about 40 seconds using an Intel Core i7-5600 CPU at 2.6 GHz. So the model can be used to optimize this type of ECB. Due to the fact that the error of the predicted flux density in the top of the pins next to the air gap increases with frequency, the model should be improved with a more detailed pin and or air gap model. Due to the field suppression in the pin, the air gap reluctance is also a function of the frequency. Therefore, the air gap and pin reluctance should be calculated in a combined FEM model as a function of the frequency and the field strength amplitude to make sure the model is also valid at speeds of about $10000 \mathrm{rpm}$.

\section{REFERENCES}

[1] W. Zimmermann, "Rechnung und versuch bei der scheibenförmigen wirbelstrombremse," Archiv für Elektrotechnik, vol. 10, no. 3-4, pp. 133-156, 1921.

[2] W. Smythe, "On eddy currents in a rotating disk," Electrical Engineering, vol. 61, no. 9, pp. 681-684, 1942.

[3] H. A. Wheeler, "Formulas for the skin effect," Proceedings of the IRE, vol. 30, no. 9, pp. 412-424, 1942.

[4] C. Holtmann, "Elektrodynamische bremse," German Patent DE102 016108 646B4, 2019

[5] M. Zec, "Theory and numerical modelling of lorentz force eddy current testing," Ph.D. dissertation, technical university ilmenau, 2012.

[6] L. Ye, G. Yang, and D. Li, "Analytical model and finite element computation of braking torque in electromagnetic retarder," Frontiers of Mechanical Engineering, vol. 9, no. 4, pp. 368-379, 2014.

[7] J. R. Nagel, "Induced eddy currents in simple conductive geometries: Mathematical formalism describes the excitation of electrical eddy currents in a time-varying magnetic field," IEEE Antennas and Propagation Magazine, vol. 60, no. 1, pp. 81-88, 2017.

[8] A. Jack and B. Mecrow, "Methods for magnetically nonlinear problems involving significant hysteresis and eddy currents," IEEE Transactions on Magnetics, vol. 26, no. 2, pp. 424-429, 1990.

[9] R. Hilzinger and W. Rodewald, Magnetic materials: fundamentals, products, properties, applications. Vacuumschmelze, 2013.

[10] O. C. Zienkiewicz, R. L. Taylor, and J. Z. Zhu, The finite element method: its basis and fundamentals. Elsevier, 2005.

[11] J. Edwards, B. Jayawant, W. Dawson, and D. Wright, "Permanentmagnet linear eddy-current brake with a non-magnetic reaction plate," IEE Proceedings-Electric Power Applications, vol. 146, no. 6, pp. 627631, 1999.

[12] I. R. Ciric, "Scalar potential formulations for magnetic fields produced by arbitrary electric current distributions in the presence of ferromagnetic bodies," IEEE transactions on magnetics, vol. 50, no. 1, pp. 1-6, 2013.

\section{BIOGRAPHIES}

Christoph Holtmann studied mechanical engineering at the University of Applied Sciences Bremen. In 2013 he specializes in thermo- and fluid dynamics at the Technical University of Ilmenau, where he received his master's degree. Since then he has been a research assistant at the German Aerospace Center (DLR), where he works on eddy current brakes.

Andreas Möckel studied electrical engineering at the Technical University Ilmenau. He worked there as a temporary, later as a permanent scientific assistant at the Institute for Electric Power and Control Engineering on the field of electrical drives and obtaining his doctorate and habilitation in the field of commutation of commutator motors. Since 2006 he leads the group of small electrical drives provisionally. The main points of his research are electronically and mechanically commutated motors in a wide performance range. 